\title{
The Effect of Head Positioning and Head Tilting on the Incidence of Intraventricular Hemorrhage in Very Preterm Infants: A Systematic Review
}

\author{
Karen A. de Bijl-Marcus ${ }^{a}$ Annemieke J. Brouwer ${ }^{a}$ Linda S. de Vries ${ }^{a}$ \\ Gerda van Wezel-Meijler ${ }^{b}$ \\ a Department of Neonatology, Wilhelmina Children's Hospital, University Medical Center Utrecht, Utrecht, and \\ ${ }^{b}$ Department of Neonatology, Isala Woman-Child Center, Zwolle, The Netherlands
}

\section{Keywords}

Intraventricular hemorrhage $\cdot$ Head position $\cdot$ Head rotation $\cdot$

Premature infant · Preterm birth · Tilting

\begin{abstract}
Background: Despite advances in neonatal intensive care, germinal matrix-intraventricular hemorrhage (GMH-IVH) remains a frequent, serious complication of premature birth. Neutral head position and head tilting have been suggested to reduce the risk of GMH-IVH in preterm infants during the first $72 \mathrm{~h}$ of life. Objective: The aim of this study was to provide a systematic review of the effect of neutral head positioning and head tilting on the incidence of GMH-IVH in very preterm infants (gestational age $\leq 30$ weeks). In addition, we reviewed their effect on cerebral hemodynamics and oxygenation. Methods: Literature was searched (June 2016) in the following electronic databases: CINAHL, Embase, Medline, SCOPUS, and several trial registers. Results: One underpowered trial studied the effect of head positioning on the incidence of GMH-IVH. This randomized controlled trial enrolled 48 preterm infants and found no effect on the occurrence of GMH-IVH. Three observational studies investigated the effect of head rotation and/or tilting on ce-
\end{abstract}

\section{KARGER}

(c) 2016 S. Karger AG, Basel

E-Mail karger@karger.com

www.karger.com/neo rebral oxygenation in 68 preterm infants in total. Their results suggest that cerebral oxygenation is not significantly affected by changes in head positioning. The effect of head positioning and/or tilting on cerebral hemodynamics was described in 2 observational studies of 28 preterm infants and found no significant effect. Conclusions: There is insufficient evidence regarding the effect of head positioning and tilting on the incidence of GMH-IVH and cerebral hemodynamics and oxygenation in preterm infants. We recommend further research in this field, especially in extremely preterm and clinically unstable infants during the first postnatal days.

(c) 2016 S. Karger AG, Basel

\section{Introduction}

Germinal matrix-intraventricular hemorrhage (GMH-IVH) is a major, frequently occurring complication of preterm birth. Of the extremely premature infants (gestational age [GA] $<28$ weeks), $20-25 \%$ will develop a GMH-IVH, with the incidence being inversely proportional to GA [1]. Typically, GMH-IVH originates from the germinal matrix, a highly vascularized collection of 
neuronal-glial precursor cells in the developing brain that involutes from about 26 weeks of gestation onwards [2]. The etiology of GMH-IVH in preterm infants is multifactorial. One key factor is the intrinsic fragility of the germinal matrix vasculature [2]. The delicate blood vessels easily rupture when rapid changes in cerebral perfusion occur. This may subsequently lead to bleeding into the ventricles (intraventricular hemorrhage). A second contributing factor is the vessel pattern of the venous system in this area. Due to the U-shaped alignment, the veins are prone to venous congestion, which can cause vessel damage and bleeding [3]. Thirdly, disturbances and fluctuations in cerebral blood flow (CBF) are common in preterm infants. Especially in sick or extremely preterm neonates, autoregulation of cerebral perfusion is impaired [4-7]. Preterm infants are thus less able to maintain a relatively constant blood flow to the brain when changes in cerebral perfusion pressure occur. Consequently, fluctuations in systemic blood pressure as well as postural changes could lead to alterations in CBF. Once a GMH-IVH has occurred, this may result in serious complications such as posthemorrhagic ventricular dilatation and periventricular hemorrhagic infarction. A large and/or complicated GMH-IVH is strongly associated with an adverse outcome, including disabilities and death $[1,3,8-10]$. Despite numerous efforts to prevent GMH-IVH in premature infants, the incidence of severe GMH-IVH has remained stable during the last few decades $[11,12]$.

Though seemingly harmless, routine caregiving events may affect cerebral perfusion and oxygenation in the preterm neonate [13]. In an attempt to avoid (rapid) fluctuations in CBF as well as intracranial pressure during routine care, several nursing interventions have been proposed. These nursing interventions are especially important during the first $72 \mathrm{~h}$ after birth, since GMH-IVH mostly develops during this time window [3, 14]. The first of these interventions consists of positioning the head of the infant in a neutral (i.e., midline) position, enabling optimal cerebral venous drainage through the internal jugular veins. The jugular veins are the major outflow paths for cranial blood. Head rotation to either side may lead to occlusion or obstruction of the jugular venous-drainage system at the ipsilateral side. Indeed, jugular phlebograms and catheterization studies in term infants and children have shown that rotating the head $90^{\circ}$ to one side may result in torsion and complete compression of the internal jugular vein on the same side [15-17]. As a consequence, hampered venous drainage could lead to venous congestion, subsequent increase in intracranial pressure, altered cerebral oxygenation, and ultimately bleeding [18-21]. The second proposed intervention consists of elevating the head of the incubator $15-30^{\circ}$ upwards (i.e., tilting) in order to facilitate venous outflow from the brain by promoting hydrostatic cerebral venous drainage [22]. A multidisciplinary focus group has identified maintaining a neutral head position together with $30^{\circ}$ tilting as 1 of 10 potential practices for the prevention of GMH-IVH. The rationale behind this recommendation was the finding that the benchmark site with the lowest rate of GMHIVH used this practice $[23,24]$.

\section{Objective}

Our aim was to provide a systematic review of studies assessing the influence of head positioning and tilting on the incidence of GMH-IVH, as well as on cerebral hemodynamics and cerebral oxygenation in preterm neonates. The latter two being important factors in the etiology of GMH-IVH. Near-infrared spectroscopy (NIRS)-monitored cerebral oxygenation, oxygen extraction, and cerebral hemodynamics are correlated to the risk of GMHIVH $[7,25]$.

\section{Methods}

Design

This systematic review was conducted according to the guidelines of the Preferred Reporting Items for Systematic Reviews and Meta-Analyses (PRISMA) [26].

\section{Criteria for Considering Studies for This Review}

Types of Studies

Randomized controlled trials (RCTs), quasi-RCTs, controlled clinical trials, as well as observational studies were eligible for inclusion. Reviews, poster presentations, conference papers, or single-case studies were excluded. Studies were eligible for inclusion if they reported an objective clinical outcome measure such as the incidence and severity of GMH-IVH, cerebral oxygenation, or cerebral hemodynamic parameters. Availability of the full text was imperative.

Types of Study Population

The target population of this review consisted of preterm neonates ( $\mathrm{GA} \leq 30$ weeks).

Types of Interventions

Studies evaluating at least 1 of the following 2 interventions for preventing GMH-IVH were included:

1. Neutral head positioning

2. Head tilting 
Types of Outcome Measures

Primary outcome:

1. Incidence of GMH-IVH diagnosed by cranial ultrasonography Secondary outcomes:

1. Cerebral perfusion

2. Cerebral oxygenation

Data Collection and Analysis

Electronic Searches. The following electronic databases were searched: Medline, Embase, CINAHL, SCOPUS, Cochrane Central Register of Controlled Trials (CENTRAL), and ClinicalTrials. gov. The search strategies are outlined in Appendix A. To avoid missing studies, we did not use a search filter to differentiate between study types. There was neither restriction on the basis of publication date nor on publication status. We restricted our search to articles written in English, Dutch, German, and French. We performed the searches on June 6, 2016.

Searching Other Resources. To identify potential additional studies, reference lists from relevant reviews and papers were hand searched.

Selection of Studies. Titles and abstracts of the search results were independently assessed by 2 review authors (K.A.d.B.-M. and A.J.B.). Full copies of all potentially relevant studies were obtained. Decision on final inclusion after retrieval of full papers was made by 2 authors independently (K.A.d.B.-M. and A.J.B.). Disagreements were resolved by discussion with a 3 rd review author (G.v.W.-M.).

Data Extraction. Two review authors extracted details of the included studies independently using a data extraction form. Any disagreements about data were resolved by consensus; if necessary a 3 rd reviewer was consulted. The following data were extracted from each study: study design, setting, patient characteristics, data collection, results, conclusion, and quality assessment.

Quality Appraisal of Individual Studies. Two authors (K.A.d.B.-M. and A.J.B.) independently evaluated the methodological quality. Discrepancies in ratings were discussed between the reviewers until consensus was reached. The methodological quality of the RCT was rated according to the "Jadad scale" (Appendix B) and the risk of bias by the "Cochrane Collaboration tool" [27]. The Jadad scale is used to independently assess the methodological quality of an RCT, with emphasis on the quality of randomization and blinding. It consists of a questionnaire composed of three questions resulting in a score ranging from 0 to 5 points. The Cochrane Collaboration risk of bias tool assesses the risk of various forms of bias (e.g. selection bias, performance bias, attrition bias, and reporting bias). Each of the items can be classified as low-risk, unclear-risk, or high-risk bias. For the quality appraisal of observational studies the "Strengthening the Reporting of Observational Studies in Epidemiology (STROBE)" statement was used [28]. The STROBE checklist is detailed in Appendix C. Each item was rated as positive, plus/ minus, or negative. To provide a final judgment about the article, scores were categorized. If less than $33 \%$ of the total score of 22 points was achieved an article was classified as "weak." If an article achieved between 34 and $66 \%$ of the total score, it was classified as "moderate." An article was classified as "strong" if the total score was higher than $66 \%$.

Grading Quality of a Body of Evidence. The quality of evidence for each of the outcome parameters was evaluated using the GRADE approach. The GRADE system entails an assessment of the quality of a body of evidence for each individual outcome spec-

Head Position and Prevention of Neonatal Intraventricular Hemorrhage ifying four levels of quality. The highest quality rating is assigned to evidence from RCTs. However, the evidence from RCTs can be downgraded depending on 5 factors. Observational studies are generally graded as "low quality", but 3 factors may increase the quality level of a body of evidence (Appendix D) [29].

\section{Results}

\section{Study Selection}

The electronic database search yielded 864 articles. One additional article was identified through hand searching. The initial selection, based on title and abstract, included 29 records that seemed to fulfill the predefined criteria. After reading the full-text articles, 24 of these 29 articles did not meet the inclusion criteria and were therefore excluded (12 reports on children/infants with a GA > 30 weeks, 7 described no relevant outcome parameters, 1 letter to the editor, 1 case report, and 3 poster abstracts). Finally, 5 studies were included in this systematic review [30-34]. Figure 1 provides a flowchart of the study selection.

\section{Study Characteristics}

The 5 included studies involved a total of 120 preterm infants undergoing positional interventions. The number of subjects ranged from 4 to 48 for each study. Three reports included only neonates with a GA $<30$ weeks $[30,33,34]$. Due to a wider variation in GA in 2 other studies, the range of GA at birth in all studies combined varied between 24 and 33 weeks. Two studies included infants with a postnatal age $\leq 72 \mathrm{~h}[30,33]$. Postnatal age in the other three studies varied considerably within, as well as between studies, ranging from $1 \mathrm{~h}$ to 3 weeks [31, $32,34]$. The designs of the included studies were predominantly prospective observational; there was 1 RCT. Table 1 gives an overview of the outcome parameters measured in the included studies. Table 2 provides an overview about the characteristics and results of the included studies.

\section{Methodological Quality}

The quality of the single RCT is presented in Table $2 \mathrm{a}$. The Jadad score was $4 / 5$, indicating adequate randomization and blinding. Evaluation of the quality of the RCT using the Cochrane Collaboration risk of bias tool indicated a low risk of bias. The results of the quality appraisal of the observational studies are presented in Table 3. The quality of the studies varied between moderate and strong. None of the studies fulfilled all 22 STROBE qual- 
Fig. 1. Flowchart of included and excluded studies. One of the 5 studies included assessed both head positioning and tilting.

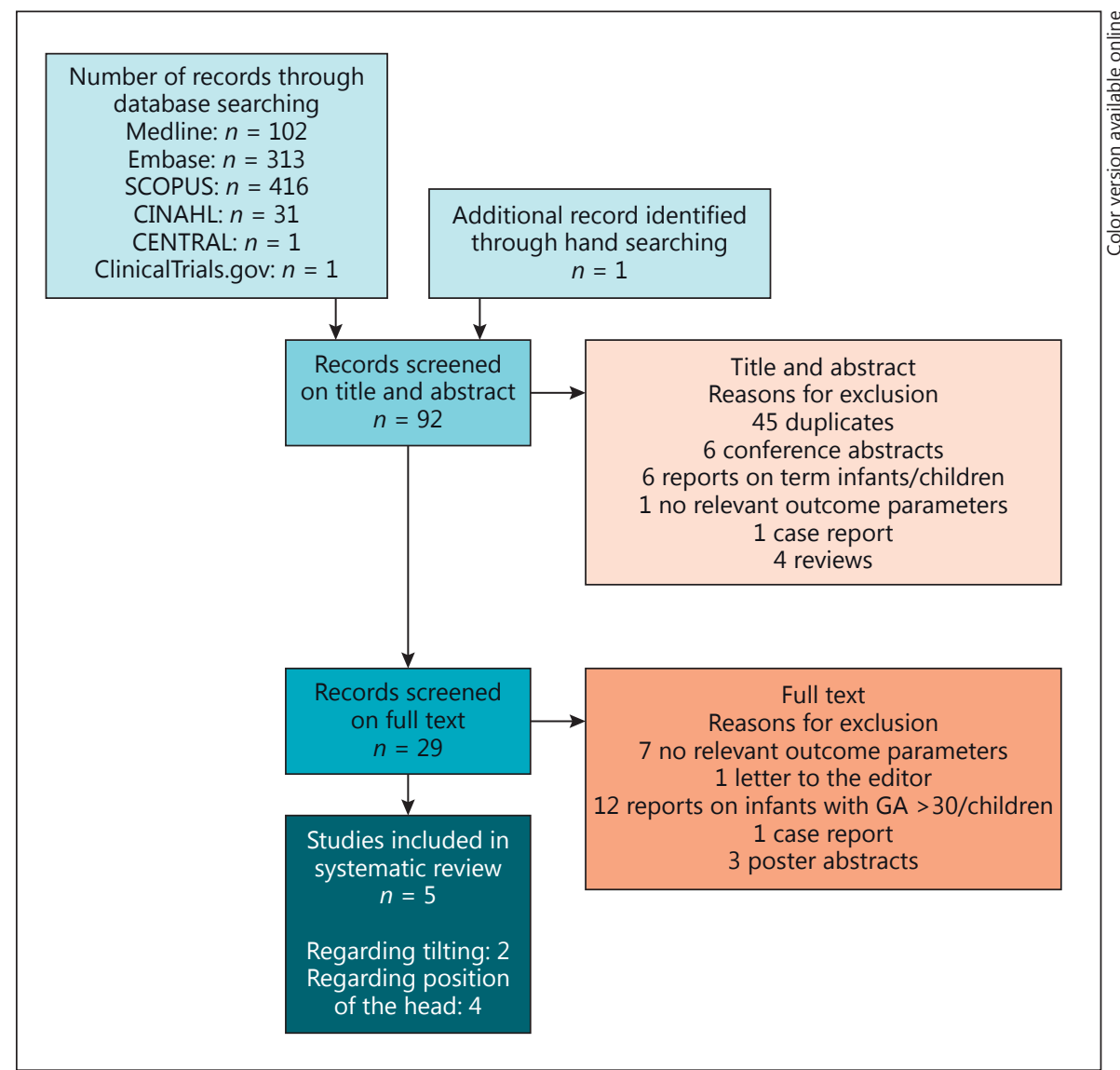

Table 1. Overview of measured outcome parameter(s) per study

\begin{tabular}{llll}
\hline First author, journal, year & $\begin{array}{l}\text { Incidence of } \\
\text { GMH-IVH }\end{array}$ & $\begin{array}{l}\text { Cerebral } \\
\text { hemodynamics }\end{array}$ & $\begin{array}{l}\text { Cerebral } \\
\text { oxygenation }\end{array}$ \\
\hline Articles regarding head position & & & $\times$ \\
Al-Abdi [30], Saudi Med J, 2011 & $\checkmark$ & $\times$ & $\checkmark$ \\
Ancora [31], Neonatology, 2010 & $\times$ & $\times$ & $\checkmark$ \\
Elser [32], Adv Neonatal Care, 2012 & $\times$ & $\times$ & $\checkmark$ \\
Liao [33], Am J Perinatol, 2015 & $\times$ & $\checkmark$ & $\checkmark$ \\
Articles regarding head tilting & $\times$ & $\checkmark$ & $\times$ \\
Ancora [31], Neonatology, 2010 & $\times$ & $\checkmark$ & $\checkmark$ \\
Buckley [34], Opt Express, 2009 & $\times$ & & $\checkmark$ \\
\hline
\end{tabular}

ity criteria. Frequently observed weaknesses were a lack of power analysis and strategies to prevent any type of bias.

\section{Study Description}

The results of the included studies are described according to the primary and secondary outcome measures: incidence of GMH-IVH, cerebral oxygenation, and cerebral hemodynamics.

\section{Incidence of GMH-IVH}

Only 1 report studied the effect of head position (neutral versus $90^{\circ}$ rotation) on the incidence of GMH-IVH. In this RCT, premature infants (mean GA 27 weeks) were 
Table 2. Table of evidence

\begin{tabular}{|c|c|c|c|c|c|}
\hline $\begin{array}{l}\text { First author, } \\
\text { year, } \\
\text { country }\end{array}$ & $\begin{array}{l}\text { Design, } \\
\text { setting, } \\
\text { parameter(s) }\end{array}$ & Participants & Results & $\begin{array}{l}\text { Quality of the RCT/ } \\
\text { observational study }\end{array}$ & $\begin{array}{l}\text { Classification of } \\
\text { evidence } \\
\text { according to } \\
\text { GRADE }\end{array}$ \\
\hline \multicolumn{6}{|c|}{ a Regarding incidence GMH-IVH } \\
\hline $\begin{array}{l}\text { Al-Abdi [30], } \\
\text { 2011, } \\
\text { Saudi Arabia }\end{array}$ & $\begin{array}{l}\text { Pilot RCT } \\
\text { NICU } \\
\text { Ultrasound } \\
\text { IVH incidence }\end{array}$ & $\begin{array}{l}n=48 \\
\text { Mean GA } 27 \text { weeks } \\
( \pm 1.3 \text { SD) } \\
\text { Intervention start: } \\
<2 \text { h after birth } \\
\text { Duration: first } 7 \text { days of } \\
\text { life } \\
90 \% \text { on mechanical } \\
\text { ventilation }\end{array}$ & $\begin{array}{l}\text { Incidence all IVH in midline (neutral) head } \\
\text { position: } 6 / 23(26 \%) \\
\text { Incidence all IVH in lateral head position } \\
\left(90^{\circ} \text { rotation): } 5 / 25(20 \%)\right. \\
\text { RR } 1.30 ; 95 \% \text { CI } 0.46-3.70 ; p=0.62 \\
\text { Incidence IVH grade III-IV } \\
\quad \text { Midline head position: } 2 / 23(9 \%) \\
\quad \text { Lateral head position: } 4 / 25(12 \%) \\
\text { RR } 1.40 ; 95 \% \text { CI } 0.61-3.37 ; p=0.94 \\
\text { Results inconclusive due to small sample size } \\
\text { (underpowered) }\end{array}$ & $\begin{array}{l}\text { (1) Jadad score: } 4 / 5 \\
\text { (2) Risk of bias } \\
\text { Random sequence: } \checkmark \\
\text { Allocation } \\
\text { concealment: } \checkmark \\
\text { Performance bias: } \times \\
\text { Detection bias: } \checkmark \\
\text { Attrition bias: } \checkmark \\
\text { Reporting bias: } \checkmark\end{array}$ & $\begin{array}{l}\text { Moderate } \\
\text { evidence } \\
\text { (minus } 1 \text { point } \\
\text { due to sparse } \\
\text { data) }\end{array}$ \\
\hline \multicolumn{6}{|c|}{ b Regarding cerebral oxygenation } \\
\hline $\begin{array}{l}\text { Ancora }[31] \\
2010 \\
\text { Italy }\end{array}$ & $\begin{array}{l}\text { Observational } \\
\text { Cross-over } \\
\text { NICU } \\
\text { NIRS } \\
\text { (TOI) }\end{array}$ & $\begin{array}{l}n=24 \\
\text { Mean GA at birth: } \\
27.5 \text { weeks }( \pm 2.8 \text { SD) } \\
\text { Mean postnatal age at the } \\
\text { time of the study: } 10.3 \text { days } \\
( \pm 7.9 \text { SD) } \\
\text { No mechanical } \\
\text { ventilation }\end{array}$ & $\begin{array}{l}\text { Midline (neutral) versus lateral } \\
\text { Horizontal midline (neutral) head position: } \\
\text { TOI } 66.7 \% \text { (SD 2.2) } \\
\text { Horizontal lateral ( } 90^{\circ} \text { rotation) head position: } 5-10 \\
\text { min after rotation: TOI } 68.1 \% \text { (SD } 2.1) \\
\text { No significant change } \\
\text { Elevated }\left(30^{\circ}\right) \text { midline (neutral) head position: } 5-10 \\
\text { min after rotation: TOI } 68.5 \% \text { (SD } 2.0) \\
\left.\text { Elevated ( } 30^{\circ}\right) \text { lateral }\left(90^{\circ} \text { rotation) head position: TOI }\right. \\
67.2 \% \text { (SD } 1.9 \text { ) } \\
\text { No significant change }\end{array}$ & $\begin{array}{l}\text { STROBE } \\
16 / 22 \\
(73 \%) \\
\text { Strong observational study }\end{array}$ & $\begin{array}{l}\text { Low evidence } \\
\text { (well-performed } \\
\text { observational } \\
\text { studies) }\end{array}$ \\
\hline & & & $\begin{array}{l}\text { Horizontal versus head tilting } \\
\text { Horizontal midline (neutral) head position: } \\
\text { TOI } 66.7 \% \text { (SD 2.2) } \\
\text { Elevated }\left(30^{\circ}\right) \text { midline (neutral) head position: } 5-10 \\
\text { min after elevation: TOI } 68.5 \% \text { (SD } 2.0) \\
\text { No significant change } \\
\text { Horizontal lateral ( } 90^{\circ} \text { rotation) head position: } \\
\text { TOI } 68.1 \% \text { (SD 2.1) } \\
\text { Elevated ( } 30^{\circ} \text { ) lateral ( } 90^{\circ} \text { rotation) head position: } 5-10 \\
\text { min after elevation: TOI } 67.2 \% \text { (SD } 1.9) \\
\text { No significant change }\end{array}$ & & \\
\hline $\begin{array}{l}\text { Elser [32], 2012, } \\
\text { USA }\end{array}$ & $\begin{array}{l}\text { Observational } \\
\text { Cross-over } \\
\text { NICU } \\
\text { NIRS } \\
\left(\mathrm{rScO}_{2}\right)\end{array}$ & $\begin{array}{l}n=24 \\
\text { GA at the time of } \\
\text { inclusion: } \\
<32 \text { weeks (mean and SD } \\
\text { not given) } \\
\text { Repeated measures } \\
\text { (days } 2,5,7 \text {, weekly } \\
\text { thereafter) } \\
\text { No information on } \\
\text { respiratory support }\end{array}$ & $\begin{array}{l}\text { Horizontal, head midline (neutral) position } \\
\text { ( } 101 \text { observations): } \\
\text { mean } \mathrm{rScO}_{2} 72.9 \% \text { (SD 9.6) } \\
\text { Horizontal, head } 45^{\circ} \text { rotated to the right } \\
\text { ( } 19 \text { observations): } \\
10-20 \text { min after rotation: mean } \mathrm{rScO}_{2} 76.0 \% \text { (SD 8.5) } \\
\text { No significant change } \\
\text { Differences in cerebral oxygenation after positional } \\
\text { change became smaller as postmenstrual age increased } \\
\text { (data not specified) }\end{array}$ & $\begin{array}{l}\text { STROBE } \\
16 / 22 \\
(73 \%) \\
\text { Strong observational study }\end{array}$ & \\
\hline $\begin{array}{l}\text { Liao [33], 2015, } \\
\text { USA }\end{array}$ & $\begin{array}{l}\text { Observational } \\
\text { Cross-over } \\
\text { NICU } \\
\text { NIRS } \\
\left(\mathrm{rScO}_{2}\right)\end{array}$ & $\begin{array}{l}n=20 \\
\text { Mean GA at birth: } \\
26.5 \text { weeks }( \pm 1.7 \mathrm{SD}) \\
\text { Mean postnatal age at the } \\
\text { time of the study: } 2 \text { days } \\
\text { (range: } 1-3 \text { ) } \\
35 \% \text { on mechanical } \\
\text { ventilation }\end{array}$ & $\begin{array}{l}\text { Elevated }\left(30^{\circ}\right) \text { midline (neutral) head position: } \\
\mathrm{rScO}_{2} 72.7 \%(\mathrm{SD} 6.4) \\
\mathrm{Elevated}^{\circ}\left(30^{\circ}\right) 45^{\circ} \text { rotated left side head position: } \\
\mathrm{rScO} 271.7 \%(\mathrm{SD} 4.8) \\
\text { (measured with sensor positioned on the left side of the } \\
\text { head } 10-20 \text { min after rotation) } \\
p<0.05 \\
\text { No statistically significant differences measured on the } \\
\text { right side of the brain and/or after rotation to the right } \\
\text { side }\end{array}$ & $\begin{array}{l}\text { STROBE } \\
20 / 22 \\
(91 \%) \\
\text { Strong observational study }\end{array}$ & \\
\hline
\end{tabular}


Table 2 (continued)

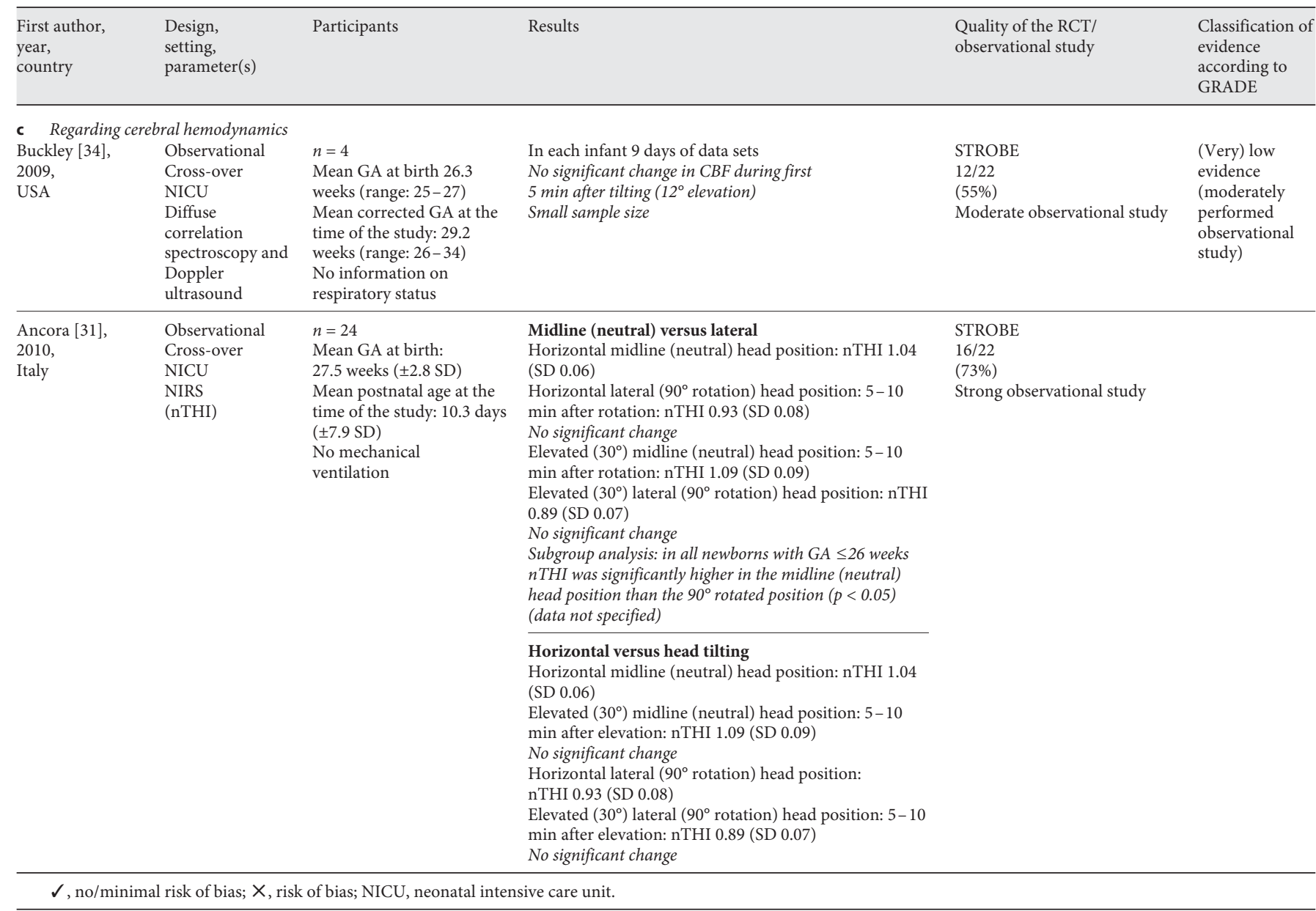

randomized to maintain either a neutral (midline) head position or a lateral head position during the first 7 days of life. Their results were inconclusive due to the small sample size (underpowered) [30]. GMH-IVH developed in $26 \%(6 / 23)$ of the infants with a neutral head position versus $20 \%(5 / 25)$ of the infants with a lateral head position (risk ratio 1.30; 95\% confidence interval 0.46-3.70; $p=0.62)$.

\section{Cerebral Oxygenation}

Three observational reports investigated the effect of head rotation on NIRS parameters. These 3 studies, including a total of 68 infants, collected the NIRS measurements at different time points following head rotation, ranging from 5 to $20 \mathrm{~min}$ after rotation. Ancora et al. [31] did not find any significant changes in the cerebral tissue oxygenation index (TOI) 5-10 min after head rotation from midline to the side. All infants were stable and none was mechanically ventilated. Elser et al. [32] found no statistically significant difference in cerebral regional oxygen saturation $\left(\mathrm{rScO}_{2}\right)$ 10-20 min after head rotation. Their results did however indicate that $\mathrm{rScO}_{2}$ changes following head rotation were smaller with increasing GA (no quantitative data presented). In a study performed by Liao et al. [33], a small but statistically significant decrease in $\mathrm{rScO}_{2}$ of $1 \%$ was found $10-20$ min after head rotation from the midline (neutral position) to the left side. Rotation to the right side did not result in a significant change [33]. The effect of tilting on cerebral oxygenation in premature infants was investigated in the study performed by Ancora et al. [31], who did not find a significant change in the cerebral TOI 5-10 min after $30^{\circ}$ elevation of the head of the incubator. 
Table 3. Overview of methodological quality of observational studies regarding head positioning and tilting

\begin{tabular}{|c|c|c|c|c|}
\hline & $\begin{array}{l}\text { Ancora } \\
{[31]}\end{array}$ & $\begin{array}{l}\text { Elser } \\
{[32]}\end{array}$ & $\begin{array}{l}\text { Liao } \\
{[33]}\end{array}$ & $\begin{array}{l}\text { Buckley } \\
{[34]}\end{array}$ \\
\hline Title/abstract & + & + & + & \pm \\
\hline Background & + & + & + & + \\
\hline Objectives & + & + & + & + \\
\hline Study design & + & + & + & + \\
\hline Setting & + & + & + & - \\
\hline Participants & + & + & + & - \\
\hline Variables & + & + & + & - \\
\hline Data sources & + & + & + & + \\
\hline Bias & - & + & + & - \\
\hline Study size & - & - & - & - \\
\hline Quantitative variables & + & + & + & + \\
\hline Statistical methods & + & - & + & + \\
\hline Participants & - & - & + & - \\
\hline Descriptive data & + & - & + & \pm \\
\hline Outcome data & + & - & + & - \\
\hline Main results & - & - & - & - \\
\hline Other analyses & - & - & + & + \\
\hline Key results & + & + & + & + \\
\hline Limitations & - & + & + & + \\
\hline Interpretation & + & + & + & + \\
\hline Generalizability & + & + & + & + \\
\hline Funding & + & + & + & + \\
\hline Total & $16 / 22$ & $15 / 22$ & $20 / 22$ & $13 / 22$ \\
\hline Category & Strong & Strong & Strong & Moderate \\
\hline
\end{tabular}

Cerebral Hemodynamics

Two observational reports on a total of 28 infants investigated the effect of head position on parameters reflecting cerebral hemodynamics. First, Ancora et al. [31] did not find any significant changes in the cerebral normalized total hemoglobin index (nTHI) 5-10 min after head rotation from the midline to the side. A subgroup analysis was conducted in infants with a GA $\leq 26$ weeks, which revealed that nTHI was significantly higher if the head was in the midline position as compared to $90^{\circ}$ rotation. No quantitative data were presented on the number of neonates in this subgroup analysis or their clinical characteristics [31]. Their study also investigated the effect of tilting on cerebral hemodynamics and found no significant change in cerebral nTHI after $30^{\circ}$ elevation of the head of the incubator [31]. Buckley et al. [34] repeatedly studied the effect of a small elevation of the head $\left(12^{\circ}\right.$ tilting $)$ in a small group of 4 infants. They did not find a significant alteration in microvascular blood flow (assessed by means of diffuse correla- tion spectroscopy) or macrovascular blood flow velocity in the middle cerebral artery (assessed by transcranial Doppler ultrasound).

\section{Grading the Quality of a Body of Evidence} Incidence of GMH-IVH

The evidence regarding the effect of head rotation on the incidence of GMH-IVH was qualified as being "moderate". Although the magnitude of the effect was investigated by a well-performed RCT, the sample size was considered too small and underpowered. Therefore, there is insufficient evidence that head rotation affects the incidence of GMH-IVH in premature infants with a GA $\leq 30$ weeks. None of the studies investigated the effect of tilting on the incidence of GMH-IVH in premature infants. Therefore, there is insufficient evidence supporting a relationship between head elevation and the occurrence of GMH-IVH.

\section{Cerebral Oxygenation}

Three well-performed observational studies provided information about changes in cerebral oxygenation after head rotation [31-33]. One of these studies also provided information on the effect of tilting on cerebral oxygenation [31]. Combined the previous studies provide low quality of evidence that head rotation and/or head tilting in premature infants (GA $\leq 30$ weeks) does not (importantly) affect cerebral oxygenation 5-20 min after rotation/elevation.

\section{Cerebral Hemodynamics}

Together the 2 observational studies represent (very) low quality of evidence that head rotation and/or tilting does not influence cerebral hemodynamics $[31,34]$.

\section{Discussion}

Our aim was to provide a systematic review of studies assessing the influence of maintaining a neutral head position and of head tilting on the incidence of GMH-IVH in very preterm neonates ( $\mathrm{GA} \leq 30$ weeks). In addition, we reviewed the effect of these postural changes on cerebral hemodynamics and oxygenation in this subset of patients, since these factors are closely related to the development of GMH-IVH [7, 25]. We found moderate quality of evidence that the incidence of GMH-IVH is not influenced by maintaining a neutral head position in very preterm infants. Al Abdi et al. [30] conducted the first and only study investigating the effect of head position on the 
occurrence of GMH-IVH in preterm infants with a GA $<30$ weeks during the first 7 days after birth. The results of this pilot study were inconclusive due to its small sample size.

Low-quality evidence indicated that there is no significant effect on cerebral oxygenation by head rotation and/ or head tilting in preterm infants [31-33]. One study performed by Liao et al. [33] revealed a small statistically significant one-sided decrease in $\mathrm{rScO}_{2}$ after head rotation to the left side. However, this decline of only $1 \%$ is unlikely to be of clinical significance.

Regarding cerebral hemodynamics, (very) low-quality of evidence showed no effect on cerebral hemodynamics after head rotation and/or head tilting [31,34].

The studies included in this systematic review were heterogeneous, especially with regard to the characteristics of the participants (e.g., variation in respiratory support), type of intervention (e.g., degree of tilting and/or rotation), and type of data collected (e.g., NIRS or ultrasound). All studies were performed in small groups of clinically stable infants. Postnatal age of most infants was more than 1 week, which is important since autoregulation of cerebral perfusion improves with postnatal age, whereas the risk of developing a GMH-IVH declines after the first postnatal days.

None of the studies reported information on the occurrence of side effects of the postural changes such as respiratory distress or increased rate of apnea. Maintaining a neutral head position hampers a prone position. Since prone position is thought to facilitate breathing it is possible that maintaining a neutral head position might predispose infants to respiratory complications [35-37].

\section{Implications for Clinical Practice}

Presently, there is insufficient evidence regarding the effect of a neutral head position and/or head tilting on the incidence of GMH-IVH in preterm infants. We therefore can neither recommend nor refute the use of a neutral head position and/or head tilting in order to prevent GMH-IVH.

\section{Implications for Research}

Further research (preferably an RCT) is needed in larger groups of preterm infants, focusing on the effect of neutral head positioning and head tilting on the incidence of GMH-IVH as well as cerebral hemodynamics and oxygenation. Special attention should be given to unstable, ill, preterm infants during the first $72 \mathrm{~h}$ after birth, since these are at greatest risk of developing a GMH-IVH. In addition, GA may influence the vulnerability to the unfavorable effects of head rotation and/or horizontal head position $[19,22,31,32]$. Therefore, extremely premature infants should be included in future research. Importantly, these future studies should include information on possible negative side effects such as increased work of breathing.

\section{Limitations and Strengths}

This review has several limitations. First, literature bias may be possible because only original, published studies were included. Results published in journals may differ systematically from those in reports, poster presentations, dissertations, or conference papers. Second, due to the lack of homogeneity among the study designs, types of measurement, and statistical analyses, it was not possible to pool the data for meta-analysis. Third, selection based on language could possibly have led to some degree of selection bias. Strengths of this review are the detailed transparent and structured data collection procedure according to the GRADE system. Second, there were no limitations set on publication date. Furthermore, two reviewers performed the selection process and the analysis of the methodological quality.

\section{Conclusion}

There is insufficient evidence regarding the effect of head positioning and/or tilting on the incidence of GMHIVH and on cerebral oxygenation and/or hemodynamics in very preterm infants. Further research is recommended with special focus on the clinically unstable, extremely preterm infant during the first $72 \mathrm{~h}$ after birth. 


\section{Appendix A}

Medline Search

(head position [tiab] OR prone [tiab] OR supine [tiab] OR head rotat* [tiab] OR neck rotat* [tiab] OR nursing [tiab] OR handling [tiab] OR positional [tiab] OR posture [tiab] OR postural [tiab] OR tilting [tiab])

AND

(“intracranial hemorrhages”[Mesh] OR “cerebral hemorrhage”[Mesh] OR intracranial hemorrhage [tiab] OR cerebral hemorrhage [tiab] OR intraventricular hemorrhage [tiab] OR intraventricular haemorrhage [tiab] OR periventricular hemorrhage [tiab] OR subependymal hemorrhage [tiab] OR intracranial bleed* [tiab] OR intraventricular bleed* [tiab] OR subependymal bleed* [tiab] OR brain hemorrhage [tiab] OR brain bleed* [tiab] OR “intracranial pressure" [Mesh] OR intracranial pressure [tiab] OR cerebral perfusion [tiab] OR brain perfusion [tiab] OR cerebral oxygen* [tiab] OR cerebral saturation [tiab] OR cerebral hemodynamic* [tiab] OR cerebral haemodynamic* [tiab] OR brain hemodynamic* [tiab] OR cerebral blood flow [tiab])

AND

("infant, premature"[Mesh] OR "infant, extremely premature"[Mesh] OR premature infant [tiab] OR neonate [tiab] OR infant [tiab] OR newborn [tiab] OR preterm [tiab] OR neonat* [tiab])

Restriction: newborn: birth - 1 month. Restriction: language: English, Dutch, German, and French

Embase Search

(prematurity/exp OR premature:ab,ti OR preterm:ab,ti OR neonatal:ab,ti OR neonate:ab,ti OR low birthweight/exp OR low birthweight:ab,ti OR infant:ab,ti OR newborn)

AND

('intracranial hemorrhage'/exp OR intracranial hemorrhage:ab,ti OR cerebral hemorrhage:ab,ti OR intraventricular hemorrhage:ab,ti OR 'brain hemorrhage'/exp OR brain hemorrhage:ab,ti OR periventricular hemorrhage:ab,ti OR subependymal hemorrhage:ab,ti OR intracranial bleeding:ab,ti OR intraventricular bleeding:ab,ti OR subependymal bleeding:ab,ti OR brain bleeding:ab,ti OR 'intracranial pressure'/exp OR intracranial pressure:ab,ti OR cerebral perfusion:ab,ti OR brain perfusion:ab,ti OR cerebral oxygenation:ab,ti OR cerebral saturation:ab,ti OR cerebral hemodynamics:ab,ti OR cerebral haemodynamics:ab,ti OR brain hemodynamics:ab,ti OR cerebral blood flow:ab,ti)

AND

(head position:ab,ti OR prone:ab,ti OR supine:ab,ti OR head rotation:ab,ti OR neck rotation:ab,ti OR head movement:ab,ti OR elevating:ab,ti OR elevation:ab,ti OR nursing:ab,ti OR handling:ab,ti OR positional:ab,ti OR posture:ab,ti OR postural:ab,ti OR tilting:ab,ti)

CINAHL Search

("infant, premature" $(\mathrm{AB})$ OR premature $(\mathrm{AB})$ OR preterm $(\mathrm{AB})$ OR neonatal $(\mathrm{AB})$ OR neonate $(\mathrm{AB})$ OR "infant, low birth weight" $(\mathrm{AB})$ OR low-birth-weight (AB) OR baby $(\mathrm{AB})$ )

AND

(intracranial hemorrhage $(\mathrm{AB})$ OR cerebral hemorrhage $(\mathrm{AB})$ OR intraventricular hemorrhage $(\mathrm{AB})$ OR brain hemorrhage $(\mathrm{AB})$ OR intraventricular bleeding $(\mathrm{AB}) \mathrm{OR}$ intracranial pressure $(\mathrm{AB})$ OR cerebral perfusion $(\mathrm{AB})$ OR brain perfusion $(\mathrm{AB}) \mathrm{OR}$ cerebral oxygenation $(\mathrm{AB})$ OR cerebral saturation $(\mathrm{AB})$ OR cerebral hemodynamics $(\mathrm{AB})$ OR cerebral blood flow $(\mathrm{AB})$ )

AND

(head position $(A B)$ OR prone $(A B)$ OR supine $(A B)$ OR head rotation $(A B)$ OR neck rotation $(A B)$ OR head movement $(A B)$ OR handling $(\mathrm{AB})$ OR positional $(\mathrm{AB})$ OR posture $(\mathrm{AB})$ OR postural $(\mathrm{AB})$ OR tilting $(\mathrm{AB})$ OR elevating $(\mathrm{AB}) \mathrm{OR}$ nursing $(\mathrm{AB})$ )

SCOPUS Search

TITLE-ABS (prematurity) OR TITLE-ABS (premature) OR TITLE-ABS (preterm) OR TITLE-ABS (neonatal) OR TITLE-ABS (neonate) OR TITLE-ABS (low birth weight) OR TITLE-ABS (infant) OR TITLE-ABS (newborn)

AND

TITLE-ABS (intracranial hemorrhage) OR TITLE-ABS (cerebral hemorrhage) OR TITLE-ABS (intraventricular hemorrhage) OR TITLE-ABS (periventricular hemorrhage) OR TITLE-ABS (subependymal hemorrhage) OR TITLE-ABS (intracranial bleeding) OR TITLE-ABS (intraventricular bleeding) OR TITLE-ABS (subependymal bleeding) OR TITLE-ABS (brain bleeding) OR TITLE-ABS (intracranial pressure) OR TITLE-ABS (cerebral perfusion) OR TITLE-ABS (brain perfusion) OR TITLE-ABS (cerebral oxygenation) OR TITLE-ABS (cerebral saturation) OR TITLE-ABS (cerebral hemodynamics) OR TITLE-ABS (brain hemodynamics) OR TITLEABS (cerebral blood flow)

AND

TITLE-ABS (head position) OR TITLE-ABS (prone) OR TITLE-ABS (supine) OR TITLE-ABS (head rotation) OR TITLE-ABS (neck rotation) OR TITLE-ABS (head movement) OR TITLE-ABS (positional) OR TITLE-ABS (posture) OR TITLE-ABS (postural) OR TITLE-ABS (tilting) 


\section{Appendix B}

Jadad Scale Scoring

Score: Assign a score of 1 point for each "yes" or 0 points for each "no"

Question 1 Was the study described as randomized (this includes the use of words such as randomly, random, and randomization)?

Question 2 Was the study described as double-blind (blinding of patients and evaluators, not necessarily therapists)?

Question 3 Was there a description of withdrawals and dropouts (explicit statement that all included patients were analyzed or the number and reasons for dropouts in all groups are given separately)?

Give 1 additional point if:

Question 1 The method to generate the randomization sequence was described and appropriate (table of random numbers, computer generated)

Question 2 The method of double blinding was described and appropriate (independent blinded assessors used, identical placebo or active placebo treatment, neither the person doing the assessment nor the study participant could identify the intervention being assessed)

Deduct 1 point if:

Question 1 The method to generate the randomization sequence was described and inappropriate (e.g., alternate allocation to groups, according to date of birth, hospital number, etc.)

Question 2 The method of double blinding was described and inappropriate (the person doing the assessment and/or the study participant could identify the intervention being assessed)

\section{Appendix C}

STROBE

Item No. Recommendation

Title and abstract $1 \quad$ (a) Indicate the study's design with a commonly used term in the title or the abstract

(b) Provide in the abstract an informative and balanced summary of what was done and what was found

Introduction

Background 2 Explain the scientific background and rationale for the investigation being reported

Objectives $\quad 3 \quad$ State specific objectives, including any prespecified hypotheses

\section{Methods}

Study design $\quad 4 \quad$ Present key elements of study design early in the paper

Setting 5 Describe the setting, locations, and relevant dates, including periods of recruitment, exposure, follow-up, and data collection

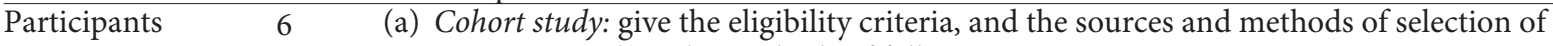

participants; describe methods of follow-up

Case-control study: give the eligibility criteria, and the sources and methods of case

ascertainment and control selection; give the rationale for the choice of cases and controls

Cross-sectional study: give the eligibility criteria, and the sources and methods of selection of participants

(b) Cohort study: for matched studies, give matching criteria and number of those exposed and unexposed

Case-control study: for matched studies, give matching criteria and the number of controls per case

\begin{tabular}{lcl}
\hline Variables & 7 & $\begin{array}{l}\text { Clearly define all outcomes, exposures, predictors, potential confounders, and effect modifiers; give } \\
\text { diagnostic criteria, if applicable }\end{array}$ \\
\hline $\begin{array}{l}\text { Data source/ } \\
\text { measurement }\end{array}$ & $8^{\text {a }}$ & $\begin{array}{l}\text { For each variable of interest, give sources of data and details of assessment (measurement); } \\
\text { describe comparability of assessment methods if there is more than 1 group }\end{array}$ \\
\hline Bias & 9 & Describe any efforts to address potential sources of bias \\
\hline Study size & 10 & Explain how the study size was arrived at \\
\hline
\end{tabular}


Item No. Recommendation

$\begin{aligned} & \text { Quantitative } \\ & \text { variables }\end{aligned}$
$\begin{aligned} & \text { Statistical } \\ & \text { methods }\end{aligned}$
were chosen and why
(a) Describe all statistical methods, including those used to control for confounding
(b) Describe any methods used to examine subgroups and interactions
(c) Explain how missing data were addressed
(d) Cohort study: if applicable, explain how loss to follow-up was addressed

Case-control study: if applicable, explain how matching of cases and controls was addressed
(e) Describe any sensitivity analyses

\section{Results}

Participants $\quad 13^{\mathrm{a}}$

Descriptive data $14^{\mathrm{a}}$

\begin{tabular}{lcl}
\hline Outcome data & $15^{\mathrm{a}}$ & $\begin{array}{l}\text { Cohort study: report numbers of outcome events or summary measures over time } \\
\text { Case-control study: report numbers in each exposure category, or summary measures of exposure } \\
\text { Cross-sectional study: report numbers of outcome events or summary measures }\end{array}$ \\
\hline Main results & 16 & $\begin{array}{l}\text { (a) Give unadjusted estimates and, if applicable, confounder-adjusted estimates and their precision (e.g., } \\
95 \% \text { CI); make clear which confounders were adjusted for and why they were included } \\
\text { (b) Report category boundaries when continuous variables were categorized } \\
\text { (c) If relevant, consider translating estimates of relative risk into absolute risk for a meaningful time } \\
\text { period }\end{array}$ \\
Other analyses & 17 & Report other analyses done - e.g., analyses of subgroups and interactions, and sensitivity analyses
\end{tabular}

(a) Report numbers of individuals at each stage of the study, e.g., numbers potentially eligible, examined for eligibility, confirmed eligible, included in the study, completing follow-up, and analyzed

(b) Give reasons for nonparticipation at each stage

(c) Consider use of a flow diagram

(a) Give characteristics of study participants (e.g., demographic, clinical, and social) and information on exposures and potential confounders

(b) Indicate number of participants with missing data for each variable of interest

(c) Cohort study: summarize follow-up time (e.g., average and total amount)

\section{Discussion}

Key results $\quad 18 \quad$ Summarize key results with reference to study objectives

\begin{tabular}{lll}
\hline Limitations & 19 & $\begin{array}{l}\text { Discuss limitations of the study (taking into account sources of potential bias or imprecision); discuss } \\
\text { and both direction and magnitude of any potential bias }\end{array}$ \\
\hline Interpretation & 20 & $\begin{array}{l}\text { Give a cautious overall interpretation of results considering objectives, limitations, multiplicity of } \\
\text { analyses, results from similar studies, and other relevant evidence }\end{array}$ \\
\hline Generalizability & 21 & \begin{tabular}{l} 
Discuss the generalizability (external validity) of the study results \\
\hline
\end{tabular}
\end{tabular}

Other information

Funding 22

22 Give the source of funding and the role of the funders for the present study and, if applicable, for the original study on which the present article is based

An Explanation and Elaboration article discusses each checklist item and gives methodological background and published examples of transparent reporting (freely available on the Web sites of PLoS Medicine [www.plosmedicine.org], Annals of Internal Medicine [www. annals.org], and Epidemiology [www.epidem.com]). Information on the STROBE Initiative is available at www.strobe-statement.org.

${ }^{a}$ Give information separately for cases and controls in case-control studies and, if applicable, for exposed and unexposed groups in cohort and cross-sectional studies. 


\section{Appendix D}

Grade System

Levels of quality of body of evidence

Quality rating

RCTs or double-upgraded observational studies

Downgraded RCTs or upgraded observational studies

High

Double-downgraded RCTs or observational studies

Moderate

Triple-downgraded RCTs, downgraded observational studies, or case series/reports

Low

Very low

Factors that may decrease the quality level of a body of evidence

1. Limitations in the design and implementation of available studies suggesting a high likelihood of bias (minus one or two levels depending on the degree of limitations)

2. Indirectness of evidence (minus one or two levels depending on the degree of uncertainty about directness)

3. Unexplained heterogeneity or inconsistency of results (minus one level)

4. Imprecision of results (wide confidence intervals) or sparse data (minus one level)

5. High probability of publication bias (minus one level)

Factors that may increase the quality level of a body of evidence

1. Large magnitude of effect (plus one level)

2. All plausible confounders would have reduced a demonstrated effect or suggest a spurious effect when results show no effect ( $p$ lus one level)

3. Dose-response gradient (plus one level)

Grade of evidence: definition

High Further research is unlikely to change the confidence in the estimate of the effect

Moderate Further research is likely to have an important impact on the confidence in the estimate of the effect and may change the estimate

Low Further research is very likely to have an important impact on the confidence in the estimate of the effect and is likely to change the estimate

Very low Any estimate of effect is very uncertain

\section{References}

1 McCrea HJ, Ment LR: The diagnosis, management and postnatal prevention of intraventricular hemorrhage in the preterm neonate. Clin Perinatol 2008;35:777-792.

2 Ballabh P: Pathogenesis and prevention of intraventricular hemorrhage. Clin Perinatol 2014;41:47-67.

3 Volpe JJ: Intracranial hemorrhage; in Volpe JJ (ed): Neurology of the Newborn, ed 5. Philadelphia, Saunders, 2008, pp 481-588.

4 Perlman JM: The relationship between systemic hemodynamic perturbations and periventricular-intraventricular hemorrhage - a historical perspective. Semin Pediatr Neurol 2009; 16:191-199.

5 Wong FY, Silas R, Hew S, Samarasinghe T, Walker AM: Cerebral oxygenation is highly sensitive to blood pressure variability in sick preterm infants. PLoS One 2012;7:e43165.

6 Wong FY, Leung TS, Austin T, Wilkinson M, Meek JH, Wyatt JS, Walker AM: Impaired autoregulation in preterm infants identified by using spatially resolved spectroscopy. Pediatrics 2008;121:e604-e611.
7 Alderliesten T, Lemmers PM, Smarius JJM, Van De Vosse RE, Baerts W, Van Bel F: Cerebral oxygenation, extraction, and autoregulation in very preterm infants who develop periintraventricular hemorrhage. J Pediatr 2013; 162:698-704

8 Morris BH, Smith KE, Swank PR, Denson SE, Landry SH: Patterns of physical and neurologic development in preterm children. J Perinatol 2002;22:31-36.

9 Pinto-Martin J, Whitaker A, Feldman J, Cnaan A, Zhao H, Rosen-Bloch J, McCulloch $\mathrm{D}$, Panet N: Special education services and school performance in a regional cohort of low-birthweight infants at age nine. Paediatr Perinat Epidemiol 2004;18:120-129.

10 Bolisetty S, Dhawan A, Abdel-Latif M, Bajuk B, Stack J, Lui K: Intraventricular hemorrhage and neurodevelopmental outcomes in extreme preterm infants. Pediatrics 2014;133: $55-62$.
11 Fanaroff AA, Stoll BJ, Wright LL, Carlo WA, Ehrenkranz RA, Stark AR, Bauer CR, Donovan EF, Korones SB, Laptook AR, Lemons JA, Oh W, Papile LA, Shankaran S, Stevenson DK, Tyson JE, Poole WK; NICHD Neonatal Research Network: Trends in neonatal morbidity and mortality for very low birthweight infants. Am J Obstet Gynecol 2007;196:147. e1-e8.

12 Jain NJ, Kruse LK, Demissie K, Khandelwal M: Impact of mode of delivery on neonatal complications: trends between 1997 and 2005. J Matern Fetal Neonatal Med 2009;22: 491-500.

13 Limperopoulos C, Gauvreau KK, O’Leary H, Moore M, Bassan H, Eichenwald EC, Soul JS, Ringer SA, DiSalvo DN, du Plessis AJ: Cerebral hemodynamic changes during intensive care of preterm infants. Pediatrics 2008; 122:e1006-e1013.

14 Perlman J, Volpe J: Intraventricular hemorrhage in premature infants. Am J Dis Child 1986;140:1122-1124.

15 Watson GH: Effect of head rotation on jugular vein blood flow. Arch Dis Child 1974;49: 237-239. 
16 Gooding CA, Stimac GK: Jugular vein obstruction caused by turning of the head. AJR Am J Roentgenol 1984;142:403-406.

17 Cowan F, Thoresen M: Changes in superior sagittal sinus blood velocities due to postural alterations and pressure on the head of the newborn infant. Pediatrics 1985;75:10381047.

18 Goldberg RN, Joshi A, Moscoso P, Castillo T: The effect of head position on intracranial pressure in the neonate. Crit Care Med 1983 11:428-430.

19 Pellicer A, Gaya F, Madero R, Quero J, Cabanas F: Noninvasive continuous monitoring of the effects of head position on brain hemodynamics in ventilated infants. Pediatrics 2002;109:434-440.

20 Emery R, Peabody JL: Head position affects intracranial pressure in newborn infants. J Pediatr 1983;103:950-953.

21 Urlesberger B, Müller W, Ritschl E, Reiterer F: The influence of head position on the intracranial pressure in preterm infants with posthemorrhagic hydrocephalus. Childs Nerv Syst 1991;7:85-87.

22 Pichler G, Boetzelar MC, Müller W, Urlesberger B: Effect of tilting on cerebral hemodynamics in preterm and term infants. Biol Neonate 2001;80:179-185.

23 Carteaux P, Cohen H, Check J, George J, McKinley P, Lewis W, Hegwood P, Whitfield JM, McLendon D, Okuno-Jones S, Kleins S, Moehring J, McConnell C: Evaluation and development of potentially better practices for the prevention of brain hemorrhage and ischemic brain injury in very low birth weight infants. Pediatrics 2003;111(4 pt 2):e489-e496.
24 Kling P: Nursing interventions to decrease the risk of periventricular-intraventricular hemorrhage. J Obstet Gynecol Neonatal Nurs 1989;18:457-464

25 Noori S, McCoy M, Anderson MP, Ramji F, Seri I: Changes in cardiac function and cerebral blood flow in relation to peri/intraventricular hemorrhage in extremely preterm infants. J Pediatr 2014;164:264-270.

26 Moher D, Liberati A, Tetzlaff J, Altman DG PRISMA Group: Preferred reporting items for systematic reviews and meta-analyses: the PRISMA statement. J Clin Epidemiol 2009; 62:1006-1012.

27 Jadad AR, Moore RA, Carroll D, Jenkinson C, Reynolds DJ, Gavaghan DJ, McQuay HJ: Assessing the quality of reports of randomized clinical trials: is blinding necessary? Control Clin Trials 1996;17:1-12.

28 Vandenbroucke JP, Von Elm E, Altman DG, Gøtzsche PC, Mulrow CD, Pocock SJ, Poole C, Schlesselman JJ, Egger M; STROBE Initiative: Strengthening the reporting of observational studies in epidemiology (STROBE): explanation and elaboration. PLoS Med 2007;4: 1628-1654.

29 Atkins D, Best D, Briss PA, Eccles M, FalckYtter Y, Flottorp S, Guyatt GH, Harbour RT, Haugh MC, Henry D, Hill S, Jaeschke R, Leng G, Liberati A, Magrini N, Mason J, Middleton P, Mrukowicz J, O'Connell D, Oxman AD, Phillips B, Schünemann HJ, Edejer T, Varonen $\mathrm{H}$, Vist GE, Williams JW Jr, Zaza S; GRADE Working Group: Grading quality of evidence and strength of recommendations. BMJ 2004;328:1490-1494.

30 Al-Abdi SY, Nojoom MS, Aishaaian HM, AlAamri MA: Pilot-randomized study on intraventricular hemorrhage with midline versus lateral head positions. Saudi Med J 2011;32: 420-421.
31 Ancora G, Maranella E, Actei A, Pierantoni L, Grandi S, Corvaglia L, Faldella G: Effect of posture on brain hemodynamics in preterm newborns not mechanically ventilated. Neonatology 2010;97:212-217.

32 Elser HE, Holditch-Davis D, Levy J, Brandon $\mathrm{DH}$ : The effects of environmental noise and infant position on cerebral oxygenation. Adv Neonatal Care 2012;12(suppl 5):S18-S27.

33 Liao SM, Rao R, Mathur AM: Head position change is not associated with acute changes in bilateral cerebral oxygenation in stable preterm infants during the first 3 days of life. Am J Perinatol 2015;32:645-652.

34 Buckley EM, Cook NM, Durduran T, Kim MN, Zhu C, Choe R, Yu G, Schultz S, Sehgal CM, Licht DJ, Arger PH, Putt ME, Hurt H, Yodh AG: Cerebral hemodynamics in preterm infants during positional intervention measured with diffuse correlation spectroscopy and transcranial Doppler ultrasound. Opt Express 2009;17:12571-12581.

35 van der Burg PS, Miedema M, de Jongh FH, Frerichs I, van Kaam AH: Changes in lung volume and ventilation following transition from invasive to noninvasive respiratory support and prone positioning in preterm infants. Pediatr Res 2015;77:484-488.

36 Balaguer A, Escribano J, Roqué M, Rivas-Fernandez M: Infant position in neonates receiving mechanical ventilation. Cochrane Database Syst Rev 2013;28:CD003668.

37 Gouna G1, Rakza T, Kuissi E, Pennaforte T, Mur S, Storme L: Positioning effects on lung function and breathing pattern in premature newborns. J Pediatr 2013;162:1133-1137. 